Article

\title{
Evaluation of a Rehabilitation System for the Elderly in a Day Care Center
}

\author{
Giuseppe Palestra ${ }^{1, *}$ (D), Mohamed Rebiai ${ }^{1}$, Estelle Courtial ${ }^{2}$ and Dimitrios Koutsouris ${ }^{3}$ \\ 1 StreamVision sas, 9 Rue Notre Dame de Lorette, 75009 Paris, France; info@streamvision.com \\ 2 Laboratory PRISME, University of Orleans, F45072 Orleans, France; estelle.courtial@univ-orleans.fr \\ 3 Biomedical Engineering Laboratory, National Technical University of Athens, 10682 Athens, Greece; \\ dkoutsou@biomed.ntua.gr \\ * Correspondence: giuseppepalestra@gmail.com
}

Received: 31 October 2018; Accepted: 15 December 2018; Published: 22 December 2018

\begin{abstract}
This paper presents a rehabilitation system based on a customizable exergame protocol to prevent falls in the elderly population. The system is based on depth sensors and exergames. The experiments carried out with several seniors, in a day care center, make it possible to evaluate the usability and the efficiency of the system. The outcomes highlight the user-friendliness, the very good usability of the developed system and the significant enhancement of the elderly in maintaining a physical activity. The performance of the postural response is improved by an average of $80 \%$.
\end{abstract}

Keywords: fall prevention; rehabilitation; exergames; depth sensor

\section{Introduction}

The average age of the global population continues to grow thanks to a longer life expectancy. The World Health Organization (WHO) calculates that between 2015 and 2050, the world's population over 60 years of age will double [1]. At the same time, the global healthcare system has to adapt to the new demographic conditions. Falls are the leading cause of accidental injury, particularly when it comes to elderly people. Around $28-35 \%$ of people, over 65 years of age, fall each year increasing to $32-42 \%$ for people over 70 years of age [2]. Falls are responsible for approximately $40 \%$ of all accidental deaths [2]. Moreover, falls represent an important economic impact that affects directly the healthcare system and indirectly individuals, family, and communities. For example, the direct healthcare system costs are \$1049 in Finland and \$3611 in Australia per one fall [1]. In order to reduce falls in elderly people physical exercises to improve strength and balance are of fundamental importance.

This study aims to present and to investigate a rehabilitation system based on a customizable exergame protocol to improve joint flexibility, coordination, balance, and muscle strength of elderly people. In this study, the main contributions are the following:

1. it proposes a new physical rehabilitation exergame protocol for the elderly;

2. the exergame protocol is multilevel and customizable according to the physical capabilities and clinical needs of the elderly by physiotherapists;

3. the system is based on low-cost depth sensors and no calibration, or training phases are required making the system feasible in home settings;

4. the proposed system was qualitatively evaluated on healthy adult volunteers;

5. the proposed system was qualitatively and quantitatively evaluated on elderly people;

6. the evaluation of the system was carried out in a real scenario;

7. long-term follow-up (six months);

8. this study provides the clinical perspectives on exergames in rehabilitation systems for the elderly. 
The rehabilitation system and the customized exergame protocol is a part of the European project called KINOPTIM project. The KINOPTIM project aims at developing an innovative system for fall prevention and rehabilitation for the elderly. This paper is structured as follows. Section 2 presents an overview of the KINOPTIM system. Section 3 provides a description of the implemented exergames and details each posture required by the system. Section 4 describes the experiments and Section 5 illustrates the experimental results. Finally, discussion and conclusion are given in Section 6.

\section{Related Work}

Recent studies showed that physical exercise programs reduce the risk of falling and improve appreciably the motor functions of the elderly [3-6] However, motivation to engage in physical exercises is scarce in the elderly [7] and new methods to engage elderly people in physical exercises should be used. Currently, new measurements technologies (portable, inexpensive, with network connectivity, and also for complex body movement [8]) are applied to the field of game and augmented reality [9]. These new devices in the context of augmented reality suggest that these tools can be used to prevent falls in the elderly population thanks to the regular monitoring of fall risk [10-13]. Physical exercise protocols carried out by using video games combining exercise, known as exergames $[14,15]$, generate a lot of interest in the field of fall prevention especially when it is based on low-cost devices such as Nintendo Wii, Microsoft Kinect, or Asus Xtion. The prevention of fall, carried out by using exergames at home, has several advantages over the conventional protocols. It makes the prevention accessible to more seniors. Exergames enable the seniors to take part of their own health management. They are motivated through an enjoyable game interface that trains motor abilities but also cognitive skills. Moreover, exergames could be implemented with different levels of difficulty, allowing each senior to begin with a comfortable level and then proceed gradually to a more difficult level [16].

In very recent years, numerous study addressed solutions relating to the use of the exergames in fall rehabilitation and prevention [17]. Smeddinck et al. [18] conducted a study of five weeks where exergames have been integrated into a traditional rehabilitation protocol. Results have indicated an increase in motivational aspects, engagement, and autonomy. The system of Brox et al. [19] proposed mini exergames that can motivate the elderly to exercise and increase their self-efficacy. They reported an increase of the balance capabilities and a significant improvement of the fun in the exergames. In [20] authors also highlighted the creation of the games can be difficult since no specific guidelines exist. Eltoukhy et al. [21] evaluated the use of the Kinect during a static single leg stand exercise and a dynamic balance tests for elderly people. Kawamoto and da Silva [22] used a depth sensors to develop games that promote quality of life among the elderly. In [23] a study aimed at determining use and perceptions of exergames during a 5-week experiment is presented. The outcomes stated that exergames are useful for improving engagement in physical activity. Ejupi et al. [3] examined the feasibility of a Kinect based fall risk system. The findings showed the feasibility of their system for fall risk assessment but the authors underlined that further investigations should be necessary. As reported in the literature, even if there is great interest in the use of exergame to improve the motor functions of the elderly, further and deeper studies are still necessary to assess the true potential of exergames addressing fall prevention and rehabilitation issues.

\section{System Overview}

The KINOPTIM system is comprised of multiple modules in order to provide a holistic fall management service. The KINOPTIM project is not a simple collection of technologies but an integration of highly innovative solutions that supports the elderly in a friendly manner. The proposed system uses a depth sensor, a RGB camera, a display, and a workstation. The system is composed of the following modules:

1. a Tele-Monitoring (TM) module;

2. a Rehabilitation and Gaming (RG) module; 
3. a Medical Business Intelligence (MBI) module.

A detailed description of the algorithms used in each module of the KINOPTIM system is out of the scope of this paper but details can be found in [24-27].

\subsection{Tele-Monitoring Module}

The TM module uses video frames from the webcam to achieve several functionalities. The first one is to evaluate the motion kinematics and the temporal evolution of the skeletal articulation structure of a senior in order to extract gait features. Two sub-modules based on image processing algorithms are necessary to extract gait features [24,25]: a detection and tracking sub-module and a gait feature extraction sub-module. The second functionality is to provide a Fall Risk Index (FRI) [26]. The FRI is used to help the physiotherapist to define a customized exergame protocol for the seniors.

\subsection{Rehabilitation and Gaming Module}

This module implements the KINOPTIM exergame protocol. Once the elderly have accessed to their individually customized exergame protocols, they play exergames and at the same time they visualize in real-time the posture performance and the score. The RG module uses depth frames to estimate the body posture of the senior and compares it to the posture required by the exergame. A score (RG_SCORE), which indicates how well a person plays an exergame, is assigned to each user at the end of the exergame [17]. The Rehabilitation and Gaming module calculates the RG_SCORE using the body posture of the participant (coordinates $x, y, z$ of each skeleton joint) and its matching (each skeleton joint) with the posture required by the exergame in respect of the exergame level chosen by the physiotherapist.

\subsection{Medical Business Intelligence Module}

The MBI module assists the physiotherapist in creating the exergame protocol for the elderly [27]. This module provides several services through a web interface called the KINOPTIM web portal. The latter is a graphical user interface that makes possible the access and the query of the MBI. The MBI contains database and data analysis functionality useful for the follow up of the training.

\section{KINOPTIM Exergame Protocol}

Implemented in the RG module, the protocol is based on exergames that engage people in an entertaining way while offering motivation for physical exercising [25]. An exergame is split into a set of static postures to be performed. The senior has to reach the desired posture in a given time defined according to the difficulty level of the exergame. The quality of the performance between the desired postures and the ones realized by the senior is scored (RG_SCORE) and ranked in stars. Stars are a graphical evaluation of the matching quality of the postures. The exergames are conceived, by the physiotherapist, to improve balance/coordination, gait, muscle strength and joint flexibility of the senior. The exergames are inspired by published literature regarding the physical activity and exercise for older adults [28-30] (http:/ / www.nhs.uk/exercises-for-older-people).

The KINOPTIM exergame protocol consists of five exercises (three kind of exercises) with different levels of difficulty: easy, medium, and hard. The five exercises are: arms extension, left knee extension, right knee extension, sideways left leg lift, and sideway right leg lift. The aim of the first exercise is to improve the arm extension. The other exercises focus on improving the flexibility of the knees and legs joints. Considering a set of multilevel exercises and according to the senior's physical condition, the physiotherapist can create a customized exergame protocol for the senior. For example, a senior, showing difficulties with moving left leg, will need to follow a protocol that does not include left leg exergames. All exercises start with a rest posture. The senior is standing in the front of the system. Feet flat on the floor and shoulder-width apart. If needed, the senior may use the back of a chair to improve their balance. 


\subsection{Extension of the Arms}

The arms extension exergame is conceived to achieve a better muscle strength and coordination. The senior slowly breathes out when he/she raises both arms to the side, keeping the shoulders as low as possible (see Figure 1). Then the senior holds the position for $5 \mathrm{~s}$ (easy level) and breathes in while slowly lowering their arms to the sides. The senior repeats this (easy level) arm movement five times. In the medium level the senior has to hold the position for $10 \mathrm{~s}$ and has to repeat the postures five times (10 times in the hard level).

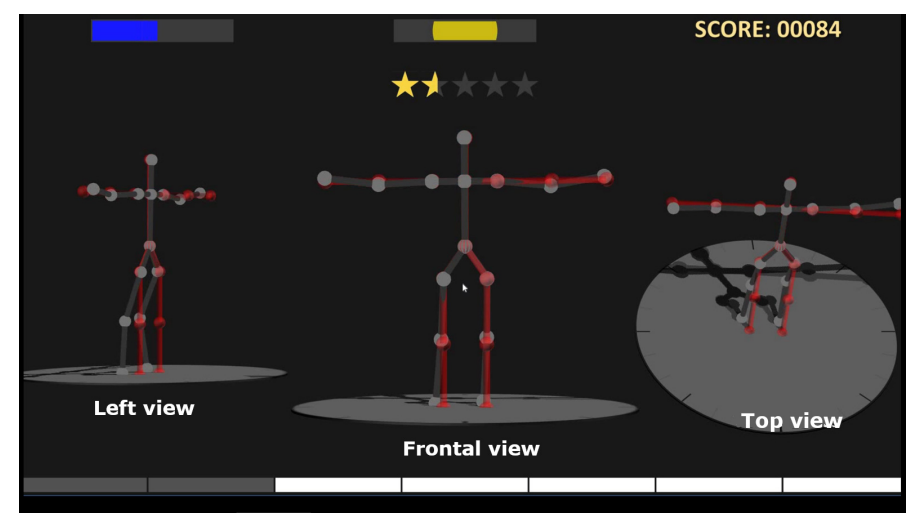

Figure 1. Arm Extension of the KINOPTIM exergame protocol. In the figure is depicted the frontal view, the lateral view, and the top view of the body posture. In red the posture of the participant and in gray the required posture.

\subsection{Extension of the Knees}

The knee extension exergame is conceived to maintain a sufficient active range of motion for the knee joint and to improve ability to stand and balance. This exergame is very important to strengthen the lower part of the body. The senior bends the (right or left) knee backward (45 degrees at the easy level, up to 90 at the hard level) and returns to the starting position (see Figure 2-Left). He/she repeats five times (easy level). In the medium level the senior has to repeat the extension 10 times 20 times in the hard level).

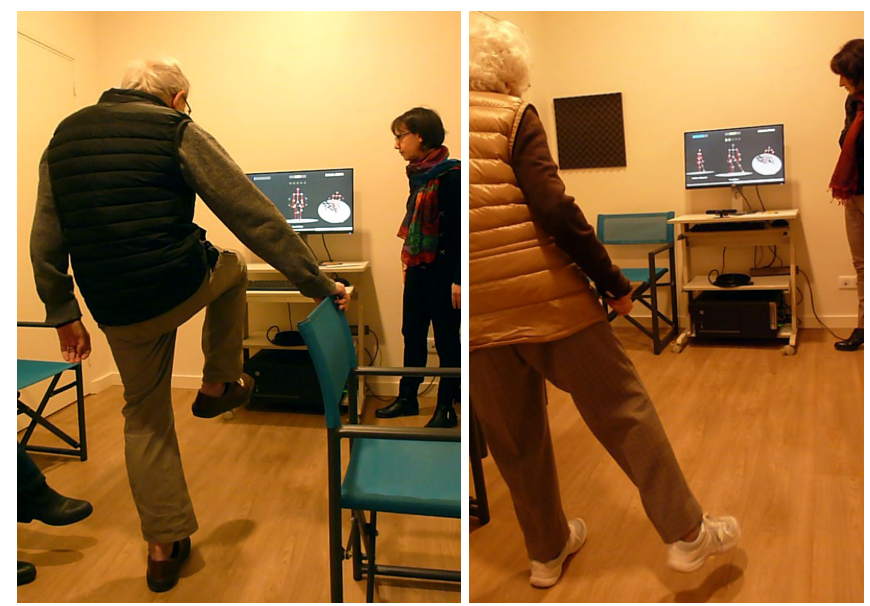

Figure 2. Overview of the KINOPTIM system during two exergames (Left: knee extension; Right: sideway right leg lift). The experimental room is equipped with technical devices.

\subsection{Sideways Lift of the Legs}

The sideways leg lift exergame is conceived to strengthen muscles, in particular quadriceps, hip flexor, and abdominals. The senior raises the (right or left) leg to the side (15 degrees at the 
easy level, up to 30 degrees at the hard level), while maintaining the back and the hips straight (see Figure 2-Right). Then, the senior returns to the starting position and repeats five times (easy level). In the medium level the senior has to repeat the lift 10 times (20 times in the hard level).

\section{Experiments}

The evaluation of the rehabilitation system consists of: (i) a qualitative pilot study with healthy adults; (ii) and a quantitative and qualitative evaluation in order to assess the usability, the interaction quality and the efficiency of the system regarding the physical activity of the elderly.

\subsection{Experimental Setup}

The experimental room is located at the "La Maison Felippa", an elderly day care center based in Paris. It is $4 \mathrm{~m}$ long, $3 \mathrm{~m}$ wide and $3 \mathrm{~m}$ high. It has been equipped with six power outlets. Lighting was adjusted according to the recommended values to ensure the proper functioning of the depth sensor. Figure 2 shows the experimental room. The posture of the senior is captured by two devices: an Asus Xtion PRO LIVE (Taipei, Taiwan) and a Full HD 1080p Logitech C920 webcam (Lausanne, Switzerland) which were respectively selected as depth sensor and RGB camera. A $27^{\prime \prime}$ LCD monitor is used to display exergames to the elderly with a good quality. The monitor also integrates speakers useful during the exergames. The workstation, a Dell Precision Tower 7910, is equipped with an Intel Dual Xeon (3.5 GHz) (Santa Clara, CA, USA), with 64 GB of RAM, 2TB of storage and a NVIDIA Quadro M4000 with 8 GB of memory as graphic card. The workstation uses the OpenNI SDK to communicate with the Asus Xtion PRO LIVE in order to grab video frames from the camera. The OpenNI SDK acquires $640 \times 480$ depth stream at $30 \mathrm{fps}$. The monitor has been placed $0.2 \mathrm{~m}$ behind the depth sensor (Asus Xtion PRO LIVE). The distance between the display and the elderly person has been fixed at $2 \mathrm{~m}$ from the depth sensor. The KINOPTIM system runs on different virtual machines of the same physical workstation using a virtual local network, therefore no Internet connection or wireless local area network is required, according to the ethical and legal guidelines of the KINOPTIM project.

\subsection{Procedure}

The experiment procedure has been submitted and approved by the French National Commission on Informatics and Liberty (CNIL) with the registered number 1967550v0. The personal data related to the elderly have been anonymized so that the individual identity can not be revealed. The anonymization provides a safeguard against accidental or mischievous release of confidential information. The name of the seniors and other personal information appeared just on the consent forms, of which one copy has been kept by the authors and the other one by the participant of the experiment.

The instructions have been written out in full in order to ensure that all the participants have the same information about the experiment. Each participant signed an informed consent, written in French (the participants' mother tongue). Participants have been asked to perform nine exergame sessions (S1, S2, S3, S4, S5, S6, S7, S8, and S9) with the rehabilitation system. Each participant attends sessions approximately every 20 days in order to avoid muscular memory effect. Participants are free to leave the experiment whenever they want. At the beginning, in the first session, two questionnaires have been administered to the participants enrolled in the experiment: the questionnaire about the Activities of Daily Living (ADL) [31] and the Nottingham Health Profile (NHP) questionnaire [32]. The 6-item Katz Index of ADL scale is used in healthcare to refer to people's dependence in daily self care activities. The Katz ADL range is 0-6, a higher score shows a greater degree of independence (6 completely independent). The NHP is designed to measure how a patient views his/her health status. It consists of 38 items dealing with physical abilities, social isolation, pain, emotional reaction, energy level and sleep. The NHP range varies from 0 (good quality of life) to 100 (worst quality). According to the ADL and NHP questionnaire results of the senior, the physiotherapist defined the exergame protocol activity. Each senior played five exergames (arms extension, knees extension (2) and sideways leg lift 
(2)) for each session at the suited level. The physiotherapist defined for all participants to use the easy level of each exergame. All the seniors participated together in a 5 min long familiarization session. The physiotherapist introduced the rehabilitation system providing a simple physical description of it. The participants were admitted one at a time in the experimental room. Once they entered the experimental room, they were placed in front of the display. Subsequently, once the elderly person felt comfortable, they started the first exergame session (S1) under the supervision of a physiotherapist or an ICT professional [17]. At the end of each session (lasted $15 \mathrm{~min}$ ), a debriefing was given to each participant. At the end of the S9, the participant answered to a questionnaire aiming at qualitatively evaluating the system (usability and interaction quality). All sessions have been performed under the supervision of a physiotherapist or an ICT professional.

\subsection{Participants}

Participants were recruited at the "La Maison Felippa" where the experiment has been carried out. Six voluntary seniors ( $n=6$, three women and three men to obtain a balanced sex group) aged 80.33 $(\mathrm{SD}=4.27)$ (for details see Table 1 ) were selected. To be included in the study, participants had to be in the range 65-85 years of age, be able to walk without any support, with no cognitive impairments and no falls episodes in the last month.

Table 1. Details on the participants involved in the experiment.

\begin{tabular}{ccccc}
\hline ID & Gender & Age & ADL Score & Falls (in the Last 12 Months) \\
\hline P1 & M & 85 & 5 & 0 \\
P2 & F & 74 & 6 & 3 \\
P3 & F & 81 & 6 & 1 \\
P4 & F & 85 & 5 & 0 \\
P5 & M & 78 & 6 & 1 \\
P6 & M & 79 & 5 & 0 \\
\hline
\end{tabular}

Participants enrolled in the experiment were administered the NHP questionnaire. The scores reached by each participant for the different parts of the NHP questionnaire are reported in Table 2.

Table 2. Participant NHP scores.

\begin{tabular}{ccccccc}
\hline & P1 & P2 & P3 & P4 & P5 & P6 \\
\hline Physical abilities (PA) & 0 & 21.15 & 21.13 & 22.9 & 0 & 0 \\
Social isolation (SI) & 0 & 0 & 34.95 & 20.43 & 40.86 & 0 \\
Pain (P) & 0 & 0 & 20.13 & 0 & 17.66 & 18.14 \\
Emotional reaction (ER) & 0 & 0 & 19.44 & 0 & 7.58 & 18.41 \\
Energy level (EL) & 0 & 0 & 0 & 0 & 0 & 0 \\
Sleep (S) & 0 & 0 & 20.36 & 0 & 0 & 46.69 \\
\hline
\end{tabular}

\section{Experimental Results}

\subsection{Pilot Study}

The elderly are not familiar with technologies such as depth sensor or exergames. These difficulties in the use of technologies lead to a preliminary pilot study. The system usability of the proposed system, according to the user-centered design methodology, has been evaluated with a formative usability test. The test consisted in carrying out two exergame sessions (S1, S2) with the system. Each session has been composed of all the five exergames of the KINOPTIM protocol. The scope of the pilot study is to collect feedback on the exergames usability from participants of this phase. The feedback provided the possibility to improve the final version of both exergames and the overall system. In order to perform the pilot experiment, users without experience in the use of exergames and depth sensor have been selected to use the system. The proposed system has been tested with 
eight persons, divided into two groups. The first one was composed by six graduate participants, the second group was composed by two healthcare professionals. All the participants were trying the system for the first time, after being properly informed, they had familiarity with IT systems and none of them had interacted earlier with depth cameras. Results showed that all participants have completed the two sessions successfully. At the end of the test, a questionnaire based on 5-point Likert scale (from 1 to 5 ) has been administered to each participant. The questions are reported below:

Q1: Overall, I am satisfied with how easy it is to use this system

Q2: It was simple to use this system

Q3: I can effectively complete my physical exercises using this system

Q4: I am able to complete my physical exercises more quickly using this system

Q5: I am able to efficiently complete my physical exercises using this system

Q6: I feel comfortable using this system

Q7: It was easy to learn to use this system

Q8: The interface of this system is pleasant

At the end of the questionnaire, a debriefing was given to each participant. Participants were asked to express their opinion about the system and to make proposal on how to improve it. All the participants confirmed that they were able to easily use the KINOPTIM system and that they felt comfortable enough. However, some improvements in terms of graphical interface have been necessary [33]. Therefore, the overall average and standard deviation scores for the system usability $(\mathrm{M}=4.06, \mathrm{SD}=0.55)$ have been calculated and can be considered as very satisfactory for the system evaluation.

\subsection{Quantitative Evaluation of the System}

In order to perform a quantitative evaluation of the system, nine experimental sessions have been performed by the elderly. Generally speaking, the system was able to operate well in the experimental environment for all the participants. The system performed all exergames without stopping and the workstation recorded all the elderly's postures correctly. To evaluate the responses of the elderly when they were interacting with the system, the focus has been put on the score (RG_SCORE) reached to perform a correct posture in a right way. The RG_SCORE reached in each exergame indicates the matching between the requested posture and the posture realized by the senior. A RG_SCORE equal to 1000 (ScoreMAX) indicates that the posture has been perfectly imitated. Conversely, a low score indicates that the exergame has been poorly-executed. With respect to the RG_SCORE, it has been observed that all the participants have increased their scores after following the KINOPTIM exergame protocol and they have improved the performance of their postural response. In Figure 3 the RG_SCORE achieved by each senior on the whole set of exergames (ALL) during nine sessions (S1, S2, S3, S4, S5, S6, S7, S8, and S9) is reported. The hypothesis is that the RG_SCORE is higher in S9 than in S1. This means that, after a repeated exposure to the system, the elderly improved their postural responses. The $t$-test was used to explore changes in the RG_SCORE from the S1 to S9 for each patient, results are reported in Table 3. In Table 4 there is a comparison of RG_SCORE in S1 and S9 for each exergame. All participants, except P2, showed a significant difference between the RG_SCORE in S1 and S9. P2 did not show a significant difference. According to healthcare professionals of the day care center, P2 was afraid of performing exergames due to the numbers of falls in the last 12 months. 


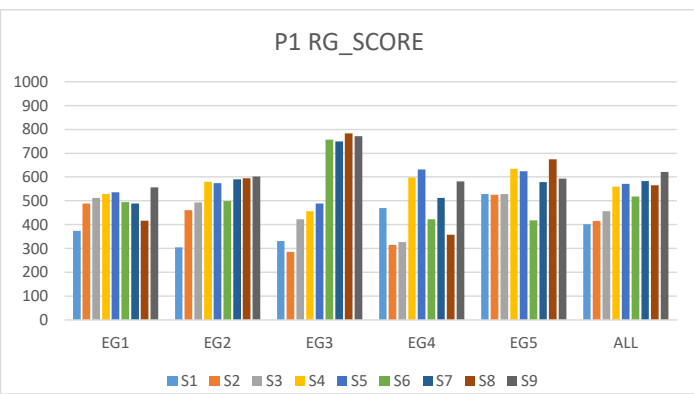

P3 RG_SCORE

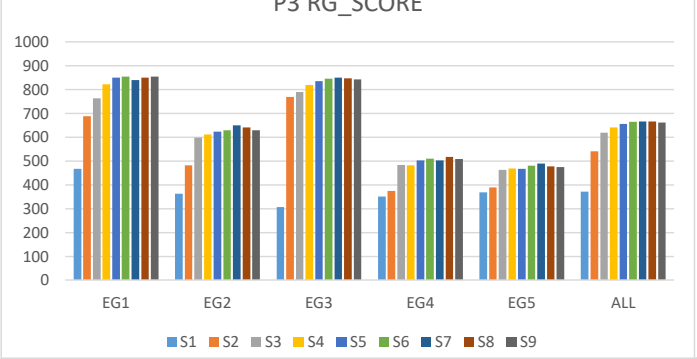

P5 RG_SCORE

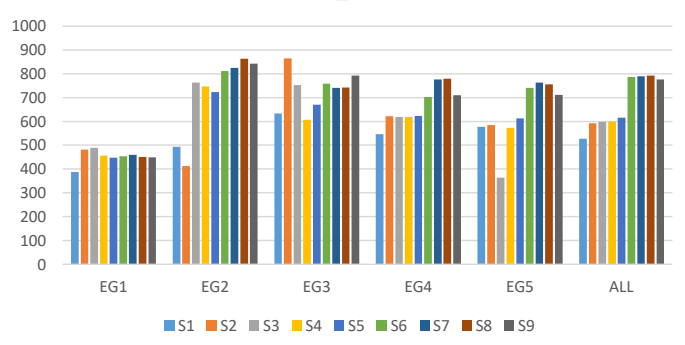

P2 RG_SCORE

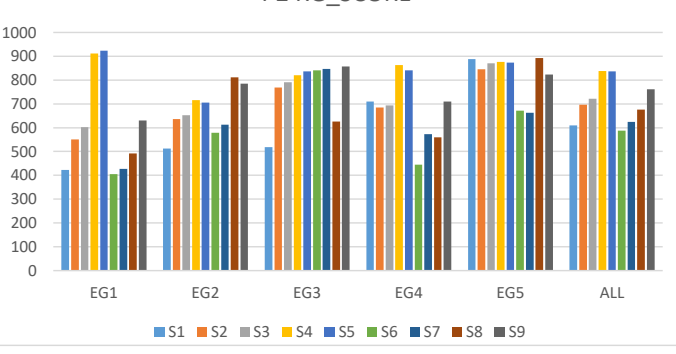

P4 RG_SCORE

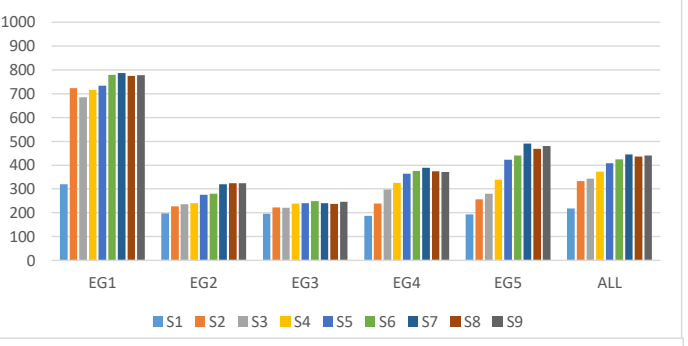

P6 RG_SCORE

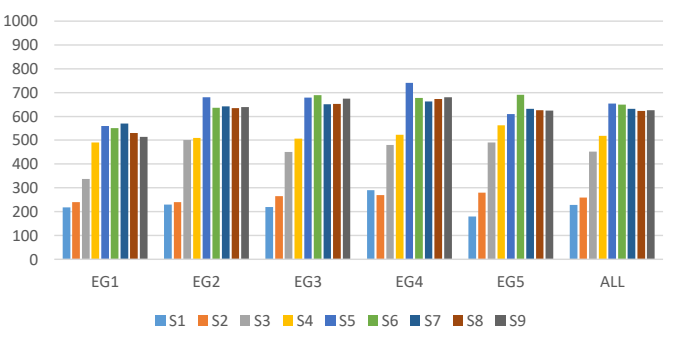

Figure 3. The graphs show the RG_SCORE obtained by each participant (P1, P2, P3, P4, P5, P6) during 5 exergames (EG1, EG2, EG3, EG4, EG5, ALL) and 9 sessions (S1, S2, S3, S4, S5, S6, S7, S8, S9) with the RG module.

Table 3. T and $p$-value from the $t$-test for RG_SCORE. Signicance levels: ${ }^{*}$ for $p<0.05,{ }^{* * *}$ for $p<0.001$

\begin{tabular}{ccc}
\hline Participant & $\boldsymbol{t}$-Test & $\boldsymbol{p}$-Value \\
\hline P1 & 3.240870 & $0.031648^{*}$ \\
P2 & 1.930274 & $0.125776^{*}$ \\
P3 & 3.727427 & $0.020342^{*}$ \\
P4 & 3.123671 & $0.035401^{*}$ \\
P5 & 3.648106 & $0.021808^{*}$ \\
P6 & 14.121438 & $0.000146^{* * *}$ \\
\hline
\end{tabular}

Table 4. Comparison of RG_SCORE in S1 and S9 for each exergame. Signicance levels: * for $p<0.05$, ** for $p<0.01,{ }^{* * *}$ for $p<0.001$

\begin{tabular}{ccc}
\hline Exergame & $t$-Test & $p$-Value \\
\hline Arm extension & -4.30733 & $0.000142^{* * *}$ \\
Knee extension & -3.61104 & $0.00238^{* *}$ \\
Sideways leg lift & -2.22908 & $0.018169^{*}$ \\
\hline
\end{tabular}

Linear regressions (see Figure 4) were used to determine the slope of improvement in RG_SCORE over six months for all participants: $\beta=0.54$ and correlation coefficient $r=0.57$ that showed a moderate correlation. 


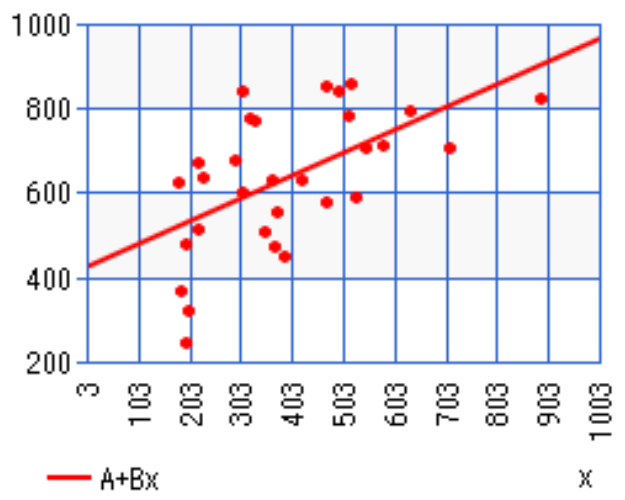

Figure 4. Linear regression for all participants and for all exergames. On the x-axe there are RG_SCORE obtained by all participants during S1 (first exergames session), RG_SCORE obtained during S9 (last exergames session) by all participants are on the y-axe.

All the participants showed an improvement in the average RG_SCORE among the sessions as reported in Table 5. Moreover, respect to the RG_SCORE, it has been observed that, in percentage, the improvement in postural response of the participants had a rate equal to $63.14 \%$ in $\mathrm{P} 1,32.12 \%$ in $\mathrm{P} 2,80.92 \%$ in $\mathrm{P} 3,95.98 \%$ in $\mathrm{P} 4,33.00 \%$ in $\mathrm{P} 5$, and $180.31 \%$ in P6. During and after six months from the end of the program none of the participants reported falls.

Table 5. RG_SCORE for each session of the whole set of exercises (ALL).

\begin{tabular}{cccccccccc}
\hline & S1 & S2 & S3 & S4 & S5 & S6 & S7 & S8 & S9 \\
\hline P1 & 401 & 414 & 456 & 559 & 570 & 518 & 583 & 565 & 621 \\
P2 & 610 & 697 & 721 & 837 & 835 & 588 & 624 & 676 & 761 \\
P3 & 371 & 540 & 619 & 641 & 656 & 664 & 666 & 666 & 662 \\
P4 & 218 & 333 & 344 & 372 & 407 & 425 & 445 & 435 & 440 \\
P5 & 527 & 592 & 597 & 600 & 615 & 786 & 789 & 793 & 776 \\
P6 & 227 & 259 & 451 & 518 & 654 & 648 & 631 & 623 & 626 \\
\hline
\end{tabular}

\subsection{Qualitative Evaluation of the System}

According to the user-centered design methodology, the usability, the user-friendliness and the interaction quality of the proposed system have been evaluated thanks to a questionnaire [34]. This questionnaire has been administered to all the participants at the end of S9. The questionnaire consisted of two parts. The first five questions aimed at evaluating the general usability, the user-friendliness of the system and the ability of the elderly to use the system in an autonomous way. The second five questions of the questionnaire was related to the evaluation of the overall quality of the human-computer interaction (HCI), considering the interest enhanced in the elderly, or the benefit of using the rehabilitation system instead of physical exercises. The questionnaire reported below consists in 10 Likert-type items, with a score ranging from 1 to 5 .

Q1: The KINOPTIM system is easy to use:

Q2: The KINOPTIM system is comfortable:

Q3: The KINOPTIM system is easy to learn:

Q4: The KINOPTIM system is efficient to complete the exercises:

Q5: The graphical interface of this system is pleasant:

Q6: The system stimulates the level of involvement:

Q7: You are interested in the KINOPTIM system: 
Q8: The system helps in the physical activity:

Q9: The system enhances physical activity:

Q10: The presence of the score helps to improve the postures:

Means and standard deviations have been used to describe the data. All the participants involved in the experiment assigned a high score to the usability items and to the interaction quality items. Therefore, the overall average and standard deviation scores for the usability $(\mathrm{M}=4.6, \mathrm{SD}=0.10)$ and for the interaction quality $(\mathrm{M}=4.9, \mathrm{SD}=0.11)$ have been calculated and can be considered as very satisfactory for the system evaluation. The answers to the questionnaire have been depicted in Figure 5.

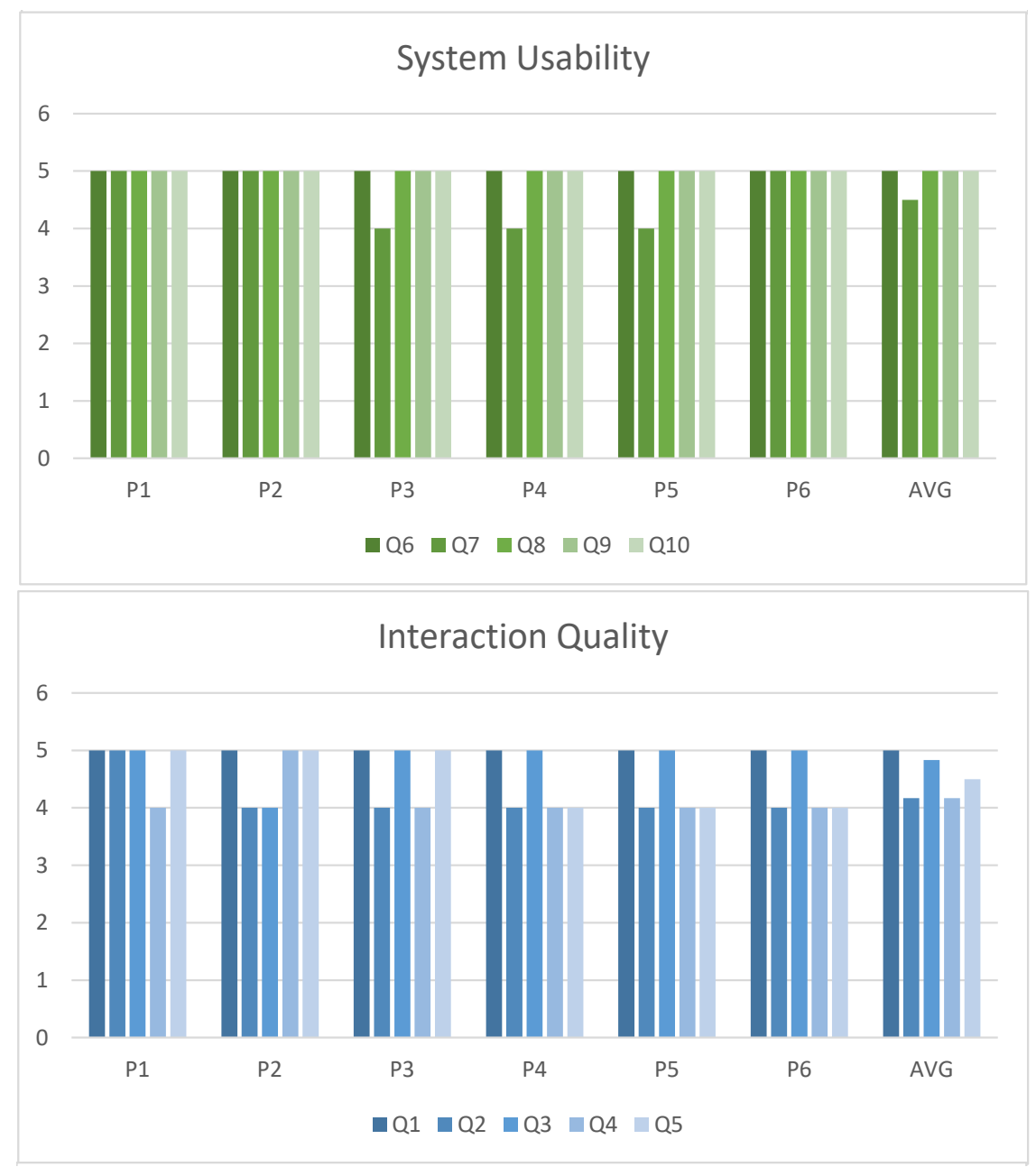

Figure 5. Results of the questionnaire for the elderly. Top: answers to the questions on general usability (first five questions). Bottom: answers to the questions on quality of interaction (second five questions).

\section{Discussion}

Although the seniors are not familiar with technologies such as depth sensors, cameras or graphical user interfaces, they found very satisfactory the usability and the quality of the interaction with the system. We excluded a quite large number of participants due to neurological and physical diseases as stated in exclusion criteria. Since the intervention was considered to be new and participants were asked to play in a supervised play to reduce the risk of injury.

Qualitative results shown that the KINOPTIM exergame protocol generates a lot of interest in the elderly and it has multiple advantages over the conventional protocols in the literature. The KINOPTIM exergame protocol: (i) enables the seniors to take part in their own health management; (ii) increases 
the seniors' motivation through an enjoyable game interface that trains motor abilities; (iii) makes the seniors focus of attention on the funny part of the rehabilitation protocol (score of the exergames) and not on the postures themselves.

Concerning the efficiency and the quantitative results of the KINOPTIM system, it was shown that all the participants have increased their scores after following the KINOPTIM exergame protocol whatever the kind of exercises (arms, knees or legs). It has been observed that the performance of the postural response, over six months training, has been improved of around $80 \%$ for each participant. All participants showed statistically significant differences between the RG_SCORE in S1 and S9. The only exception is for P2 during leg exercises because according to the physiotherapist her falling episodes in the last 12 months and her fear during the exergames. The progressive improvements in the RG_SCORE over the six month period may be due to a gain in experience with the exergames. During and after six months from the end of the training none of the participants reported falls.

Due to the great difference between studies on exergames in literature in terms of methodology, intervention protocols and measures, it is pretty difficult to compare our results to previous work. However, in [35] knees extension exergames did not reach a statistically significant improvement whereas our system was able to show a statistically significant improvement. In [36] two exergames, one for the arms and one for the legs, have been developed, as in our study, but nothing statistically significant results have been presented. In [37] experiments on leg lift exergame provide significant improvements for the right and the left leg, but no results are provided on knees or arms exergames as in our study. Sato et al. [38] showed leg muscles improvement but they did not provide any improvement in upper-body and arms exergames training as in our study. In [39] results indicated that arms gesture to initialize the tracker module is too complex for the elderly wheres in our study arm extension exergame is correctly performed since the beginning (S1). In the exergames used in the study of Konstantinidis et al. [19] outcomes showed increased significantly balance capabilities but there are not details on legs or keens extension improvements.

\section{Conclusions}

The use of new and low-cost technologies that allow the implementation of exegames for the elderly population is spreading worldwide. In the context of fall prevention for the elderly, the KINOPTIM system developed in this study proposed an efficient solution based on a customized exergame protocol that can be applied in a range of therapeutic environments. The system does not require complex materials, extended lengths of time for administration and it is low-cost. The KINOPTIM exergame protocol is defined by the physiotherapist using multilevel exercises according to the elderly's physical status. The system has been deployed in a real context: an elderly day care center in Paris. This study suggests that it is safe and feasible for the elderly to participate in a 6-month exergame protocol using a low cost device and the KINOPTIM system. We were able to detail information on each exergame session: date, time, duration, and score of exergames. These data are inaccessible from other commercial devices that cause problems with data analysis. In future investigations, we will propose to the physiotherapist to customize dynamically the level of the exergame. Globally, the rehabilitation system encountered a great success in the elderly both for its user-friendliness and for its efficiency in maintaining a physical activity. Further investigations will be extended involving a greater number of seniors in order to establish the efficiency of the rehabilitation system by comparing the obtained performance with a control group. An important point will also be to create and evaluate empathic behavior of the participants [40,41]. In order to achieve better qualitative and quantitative scientific evidences, it is necessary that future studies use statistical analyses and sample size calculations to appreciate minimal clinically important difference.

Author Contributions: Conceptualization, D.K., E.C., M.R. and G.P.; methodology, M.R., D.K.; validation, G.P. and E.C.; formal analysis, G.P., M.R. and E.C.; writing-original draft preparation, G.P.; writing-review and editing, E.C., M.R.; supervision, D.K, E.C., M.R.

Funding: This research was partially supported by a Marie Curie IAPP program, FP7-People-IAPP, Grant 324491. 
Acknowledgments: The authors would like to thank the "La Maison Felippa" a day care center for the elderly located in Paris (France).

Conflicts of Interest: The authors declare no conflict of interest.

\section{References}

1. World Health Organization. Fact Sheet 404 September 2015. 2015. Available online: http://www.who.int/ mediacentre/factsheets/fs404/en/ (accessed on 16 January 2017).

2. World Health Organization. WHO Global Report on Falls Prevention in Older Age; World Health Organization: Geneva, Switzerland, 2008.

3. Ejupi, A.; Gschwind, Y.J.; Valenzuela, T.; Lord, S.R.; Delbaere, K. A kinect and inertial sensor-based system for the self-assessment of fall risk: A home-based study in older people. Hum. Comput. Interact. 2016, 31, 261-293. [CrossRef]

4. Choi, S.D.; Guo, L.; Kang, D.; Xiong, S. Exergame technology and interactive interventions for elderly fall prevention: A systematic literature review. Appl. Ergonom. 2016, 65, 570-581. [CrossRef] [PubMed]

5. Byrne, C.A.; Collier, R.; O'Hare, G.M.P. A Review and Classification of Assisted Living Systems. Information 2018, 9, 182. [CrossRef]

6. Heinz, M.; Cho, J.; Kelly, N.; Martin, P.; Wong, J.; Franke, W.; Hsieh, W.H.; Blaser, J. The Potential of Three Computer-Based Communication Activities for Supporting Older Adult Independent Living. Information 2016, 7, 26. [CrossRef]

7. Meekes, W.; Stanmore, E.K. Motivational Determinants of Exergame Participation for Older People in Assisted Living Facilities: Mixed-Methods Study. J. Med. Internet Res. 2017, 19, e238. [CrossRef] [PubMed]

8. Civita, A.; Fiori, S.; Romani, G. A Mobile Acquisition System and a Method for Hips Sway Fluency Assessment. Information 2018, 9, 321. [CrossRef]

9. Konstantinidis, E.; Billis, A.; Paraskevopoulos, I.T.; Bamidis, P. The interplay between IoT and serious games towards personalised healthcare. In Proceedings of the IEEE 9th International Conference on Virtual Worlds and Games for Serious Applications (VS-Games), Athens, Greece, 6-8 September 2017; pp. 249-252.

10. Bieryla, K.A.; Dold, N.M. Feasibility of Wii Fit training to improve clinical measures of balance in older adults. Clin. Interv. Aging 2013, 8, 775-781. [CrossRef] [PubMed]

11. Madhushri, P.; Dzhagaryan, A.; Jovanov, E.; Milenkovic, A. An mHealth Tool Suite for Mobility Assessment. Information 2016, 7, 47. [CrossRef]

12. Birt, J.; Stromberga, Z.; Cowling, M.; Moro, C. Mobile Mixed Reality for Experiential Learning and Simulation in Medical and Health Sciences Education. Information 2018, 9, 31. [CrossRef]

13. Mittelstadt, B. Designing the Health-related Internet of Things: Ethical Principles and Guidelines. Information 2017, 8, 77. [CrossRef]

14. Mortazavi, B.; Nyamathi, S.; Lee, S.I.; Wilkerson, T.; Ghasemzadeh, H.; Sarrafzadeh, M. Near-realistic mobile exergames with wireless wearable sensors. IEEE J. Biomed. Health Inform. 2014, 18, 449-456. [CrossRef] [PubMed]

15. Morando, M.; Ponte, S.; Ferrara, E.; Dellepiane, S. Definition of Motion and Biophysical Indicators for Home-Based Rehabilitation through Serious Games. Information 2018, 9, 105. [CrossRef]

16. Lauzé, M.; Martel, D.; Agnoux, A.; Sirois, M.J.; Émond, M.; Daoust, R.; Aubertin-Leheudre, M. Feasibility, acceptability and effects of a home-based exercise program using a gerontechnology on physical capacities after a minor injury in community-living older adults: A pilot study. J. Nutr. Health Aging 2018, 22, 16-25. [CrossRef] [PubMed]

17. Courtial, E.; Palestra, G.; Rebiai, M. A Tailored Serious Game for Preventing Falls of the Elderly. In Proceedings of the International Conference on Augmented Reality, Virtual Reality and Computer Graphics, Ugento, Italy, 12-15 June 2017; Springer: Berlin, Germany, 2017; pp. 230-239.

18. Smeddinck, J.D.; Herrlich, M.; Malaka, R. Exergames for physiotherapy and rehabilitation: A medium-term situated study of motivational aspects and impact on functional reach. In Proceedings of the 33rd Annual ACM Conference on Human Factors in Computing Systems, Seoul, Korea, 18-23 April 2015; pp. 4143-4146. 
19. Brox, E.; Konstantinidis, S.T.; Evertsen, G.; Fernandez-Luque, L.; Remartinez, A.; Oesch, P.; Civit, A. Gameup: Exergames for mobility-A project to keep elderly active. In Proceedings of the XIV Mediterranean Conference on Medical and Biological Engineering and Computing, Paphos, Cyprus, 31 March-2 April 2016; Springer: Berlin, Germany, 2016; pp. 1225-1230.

20. Brox, E.; Konstantinidis, S.T.; Evertsen, G. User-Centered Design of Serious Games for Older Adults Following 3 Years of Experience With Exergames for Seniors: A Study Design. JMIR Serious Games 2017, 5, e2. [CrossRef] [PubMed]

21. Eltoukhy, M.A.; Kuenze, C.; Oh, J.; Signorile, J.F. Validation of static and dynamic balance assessment using Microsoft Kinect for young and elderly populations. IEEE J. Biomed. Health Inform. 2018, 22, 147-153. [CrossRef] [PubMed]

22. Kawamoto, A.L.S.; da Silva, F.S.C. Depth-Sensor Applications for the Elderly: A Viable Option to Promote a Better Quality of Life. IEEE Consum. Electron. Mag. 2018, 7, 47-56. [CrossRef]

23. Bird, M.; Clark, B.; Millar, J.; Whetton, S.; Smith, S. Exposure to 'Exergames' Increases Older Adults' Perception of the Usefulness of Technology for Improving Health and Physical Activity: A Pilot Study. JMIR Serious Games 2015, 3, e8. [CrossRef] [PubMed]

24. Barelle, C.; Courtial, E.; Vellidou, E.; Tsirbas, H.; Tagaris, T.; Ibanez, F.; Sanchez, E.; Koutsouris, D. Tele-monitoring and diagnostic for fall prevention: The KINOPTIM concept. In Proceedings of the IEEE-EMBS International Conference on Biomedical and Health Informatics (BHI), Valencia, Spain, 1-4 June 2014; pp. 342-345.

25. Barelle, C.; Tsirbas, C.; Ibanez, F.; Vellidou, E.; Tagaris, T.; Koutsouri, G.; Koutsouris, D. KINOPTIM: A Tele-rehabilitation gaming Platform for Fall Prevention in the Elderly Community. Int. J. Health Res. Innov. 2014, 2, 37-49.

26. Courtial, E.; Brulin, D. A Decision Support System for Preventing Falls in Elderly People. In Proceedings of the 5th EAI International Conference on Wireless Mobile Communication and Healthcare, London, UK, 14-16 October 2015; pp. 108-112.

27. Kouris, I.; Tsirbas, C.; Tagaris, T.; Vellidou, E.; Vartholomeos, P.; Rizou, S.; Koutsouris, D. KINOPTIM: The medical business intelligence module for fall prevention of the elderly. In Proceedings of the IEEE 15th International Conference on Bioinformatics and Bioengineering (BIBE), Belgrade, Serbia, 2-4 November 2015; pp. 1-4.

28. Cavanagh, P.; Evans, J.; Fiatarone, M.; Hagberg, J.; McAuley, E.; Startzell, J. Exercise and physical activity for older adults. Med. Sci. Sports Exerc. 1998, 30, 1-29.

29. Chodzko-Zajko, W.J.; Proctor, D.N.; Singh, M.A.F.; Minson, C.T.; Nigg, C.R.; Salem, G.J.; Skinner, J.S. Exercise and physical activity for older adults. Med. Sci. Sports Exerc. 2009, 41, 1510-1530. [CrossRef]

30. Asakawa, Y.; Ikezoe, T.; Hazaki, K.; Kawano, I.; Irie, S.; Kanzaki, H.; Aoki, N. Relationship between falls and knee extension strength in the elderly. J. Phys. Ther. Sci. 2001, 8, 45-48. [CrossRef]

31. Katz, S.; Downs, T.D.; Cash, H.R.; Grotz, R.C. Progress in development of the index of ADL. Gerontologist 1970, 10, 20-30. [CrossRef] [PubMed]

32. Hunt, S.M.; McKenna, S.P.; McEwen, J.; Williams, J.; Papp, E. The Nottingham Health Profile: Subjective health status and medical consultations. Soc. Sci. Med. A Med. Psychol. Med. Sociol. 1981, 15, 221-229. [CrossRef]

33. Palestra, G.; Rebiai, M.; Courtial, E.; Giokas, K.; Koutsouris, D. A Fall Prevention System for the Elderly: Preliminary Results. In Proceedings of the IEEE 30th International Symposium on Computer-Based Medical Systems (CBMS), Thessaloniki, Greece, 22-24 June 2017; pp. 550-551.

34. Rusu, C.; Rusu, V.; Roncagliolo, S.; González, C. Usability and user experience: what should we care about? Int. J. Inf. Technol. Syst. Approach (IJITSA) 2015, 8, 1-12. [CrossRef]

35. Gschwind, Y.J.; Schoene, D.; Lord, S.R.; Ejupi, A.; Valenzuela, T.; Aal, K.; Woodbury, A.; Delbaere, K. The effect of sensor-based exercise at home on functional performance associated with fall risk in older people-A comparison of two exergame interventions. Eur. Rev. Aging Phys. Act. 2015, 12, 11. [CrossRef] [PubMed]

36. Ng, P.Y.; Ng, C.K.; Al-Haddad, S.A.R. Physiotherapy for Elderly through Exergames using Microsoft Kinect Sensor. Proc. Asia-Pac. Adv. Netw. 2015, 40, 119-129. [CrossRef] 
37. Gioftsidou, A.; Vernadakis, N.; Malliou, P.; Batzios, S.; Sofokleous, P.; Antoniou, P.; Kouli, O.; Tsapralis, K.; Godolias, G. Typical balance exercises or exergames for balance improvement? J. Back Musculoskelet. Rehabil. 2013, 26, 299-305. [CrossRef] [PubMed]

38. Sato, K.; Kuroki, K.; Saiki, S.; Nagatomi, R. The effects of exercise intervention using KinectTM on healthy elderly individuals: A quasi-experimental study. Open J. Ther. Rehabil. 2014, 2, 38. [CrossRef]

39. Planinc, R.; Nake, I.; Kampel, M. Exergame design guidelines for enhancing elderly's physical and social activities. In Proceedings of the Third International Conference on Ambient Computing, Applications, Services and Technologies (AMBIENT 2013), Porto, Portugal, 29 September-3 October 2013; pp. 58-63.

40. Bamidis, P.; Gabarron, E.; Hors-Fraile, S.; Konstantinidis, E.; Konstantinidis, S.; Rivera, O. Gamification and behavioral change: Techniques for health social media. In Participatory Health Through Social Media; Elsevier: Amsterdam, The Netherlands, 2016; pp. 112-135.

41. De Carolis, B.; Ferilli, S.; Palestra, G. Simulating empathic behavior in a social assistive robot. Multimed. Tools Appl. 2017, 76, 5073-5094. [CrossRef]

(C) 2018 by the authors. Licensee MDPI, Basel, Switzerland. This article is an open access article distributed under the terms and conditions of the Creative Commons Attribution (CC BY) license (http:// creativecommons.org/licenses/by/4.0/). 